\title{
Quantum interference effects on the intensity of the $G$ modes in double-walled carbon nanotubes
}

\author{
H. N. Tran, ${ }^{1}$ J.-C. Blancon, ${ }^{2}$ R. Arenal,,${ }^{3,4}$ R. Parret,${ }^{1}$ A. A. Zahab,${ }^{1}$ A. Ayari, ${ }^{2}$ F. Vallée,${ }^{2}$ N. Del Fatti, ${ }^{2}$ \\ J.-L. Sauvajol, ${ }^{1}$ and M. Paillet ${ }^{1}$ \\ ${ }^{1}$ Laboratoire Charles Coulomb (L2C), Univ. Montpellier, CNRS, Montpellier, France \\ ${ }^{2}$ Institut Lumière Matière, (UMR5306), Université Lyon 1, CNRS, Université de Lyon F-69622 Villeurbanne, France \\ ${ }^{3}$ Laboratorio de Microscopías Avanzadas, Instituto de Nanociencia de Aragón, Universidad de Zaragoza, 50018 Zaragoza, Spain \\ ${ }^{4}$ ARAID Foundation, 50018 Zaragoza, Spain \\ (Received 8 March 2017; published 8 May 2017)
}

\begin{abstract}
The effects of quantum interferences on the excitation dependence of the intensity of $G$ modes have been investigated on single-walled carbon nanotubes [Duque et al., Phys. Rev. Lett. 108, 117404 (2012)]. In this work, by combining optical absorption spectroscopy and Raman scattering on individual index identified double-walled carbon nanotubes, we examine the experimental excitation dependence of the intensity of longitudinal optical and transverse optical $G$ modes of the constituent inner and outer single-walled carbon nanotubes. The observed striking dependencies are understood in terms of quantum interference effects. Considering such effects, the excitation dependence of the different components of the $G$ modes permits us to unambiguously assign each of them as originating from the longitudinal or transverse $G$ modes of inner and outer tubes.
\end{abstract}

DOI: 10.1103/PhysRevB.95.205411

\section{INTRODUCTION}

Single-walled carbon nanotubes (SWNTs) represent one of the best known 1D materials for studying electronic and optical phenomena such as exciton photophysics (for a review, see Ref. [1] and references therein). The features of the excitonic transitions, namely narrow and intense, make SWNTs an ideal system for exploring the effect of quantum interferences in the Raman response from energetically close optical transitions. In the presence of two closely spaced transitions, different Raman matrix elements related to different excited states, i.e., different optical transitions, contribute to the Raman response. A main consequence of quantum interferences can be to significantly distort the resonance excitation profile (REP) of a Raman active mode and consequently to complicate the extraction of the transition energies $E_{i i}$ and the corresponding damping parameters $\Gamma_{i i}$ derived from it. Hereafter, we denote the $E_{i i}$ transition for individual semiconducting and metallic nanotubes by $S_{i i}$ and $M_{i i}$, respectively $(i i=11,22,33 \ldots$ ); $M_{i i-}$ and $M_{i i+}$ represent the lower and upper components of the $M_{i i}$ transition of a metallic SWNT [2]. As evidenced by Bussi et al. [3], due to the proximity of $M_{i i-}$ and $M_{i i+}$ transitions, quantum interferences effects can have a profound consequence on the REP of the radial-breathing mode (RBM) in metallic SWNTs. It was established that neglecting interferences can lead to systematic errors in the estimates of the features of the optical transitions [3]. On the other hand, due to the proximity of two consecutive high-order excitonic transitions in semiconducting SWNTs, namely $S_{i i}$ and $S_{i+1 i+1}$ (such as $S_{33}$ and $S_{44}, S_{44}$ and $S_{55}, \ldots$ ), or to the proximity of the $S_{i+1 i+1}$ transition with $S_{i i}+E_{G}$, where $E_{G}$ is the energy of the $G$ mode, quantum interference effects were unambiguously demonstrated in the REPs of the longitudinal (LO) and transverse optical (TO) $G$ modes [4]. Especially, such quantum interference effects lead to a drastic and anomalous dependence of the relative intensity of the LO and TO $G$ mode on the laser excitation energy (see Fig. 3 of Ref. [4]). Finally, it has to be emphasized that, due to the weak dependence of the $G$ modes on the nanotube $(n, m)$ chirality, such quantum interference effects can be only evidenced from experiments performed on samples containing a single chirality, either (i) enriched species in a single $(n, m)$ SWNT or (ii) individual and isolated SWNTs.

Assuming two close excitonic transitions, namely $E_{1}$ and $E_{2}$, simplified expressions of the Raman intensity can be derived [5]. In the absence of quantum interference effect, Raman intensity takes the following form:

$$
\begin{aligned}
I\left(E_{L}\right) \propto & \left|\frac{M_{1}}{E_{L}-E_{1}+j \Gamma_{1}}-\frac{M_{1}}{E_{L}-E_{1}-E_{p h}+j \Gamma_{1}}\right|^{2} \\
& +\left|\frac{M_{2}}{E_{L}-E_{2}+j \Gamma_{2}}-\frac{M_{2}}{E_{L}-E_{2}-E_{p h}+j \Gamma_{2}}\right|^{2} .
\end{aligned}
$$

By taking into account interference effects, Raman intensity is expressed as:

$$
\begin{aligned}
I\left(E_{L}\right) \propto & \mid \frac{M_{1}}{E_{L}-E_{1}+j \Gamma_{1}}-\frac{M_{1}}{E_{L}-E_{1}-E_{p h}+j \Gamma_{1}} \\
& +\frac{M_{2}}{E_{L}-E_{2}+j \Gamma_{2}}-\left.\frac{M_{2}}{E_{L}-E_{2}-E_{p h}+j \Gamma_{2}}\right|^{2} .
\end{aligned}
$$

In both expressions, $M_{1}\left(M_{2}\right)$ condenses the electron-phonon and electron-photon matrix elements involved in the resonance with the $E_{1}=E_{i i}\left(E_{2}=E_{i+1 i+1}\right)$ transition. $\Gamma_{1}$ and $\Gamma_{2}$ are the damping parameters of the $E_{1}$ and $E_{2}$ transitions, respectively. $E_{L}$ is the laser excitation energy.

The simplified expression (2) permits us to easily simulate the effects of quantum interferences on the intensity dependence of the LO and TO $G$ modes as the function of the laser excitation. The plots of Figs. 1(a) and 1(b) illustrate the interference effects on the Raman intensity of the LO and TO $G$ modes, respectively. In these simulations, $\left|M_{1}\right|=\left|M_{2}\right|$ and the relative sign of the $M_{1}$ and $M_{2}$ matrix elements are derived from the calculations of V. N. Popov and collaborators who 
(a)

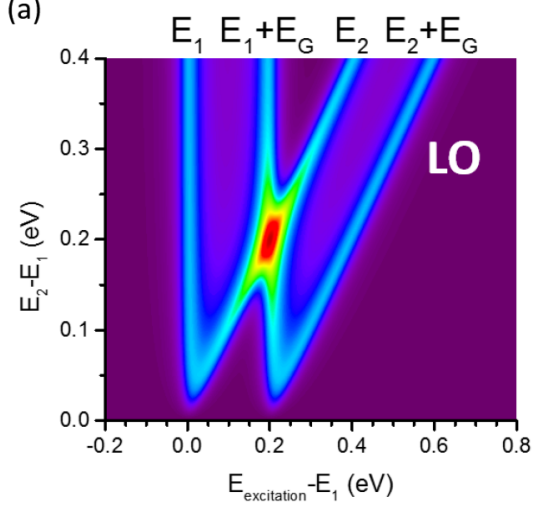

(b)

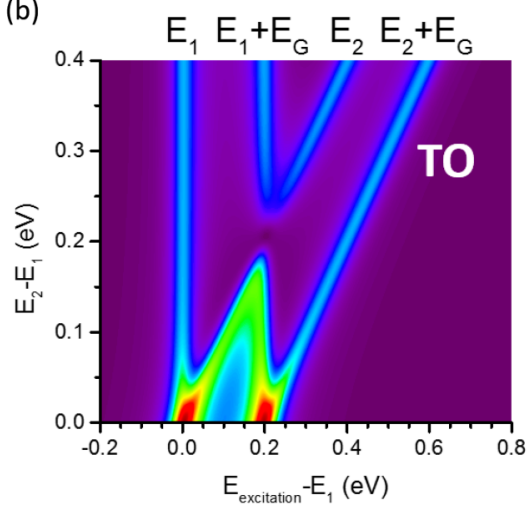

(c)

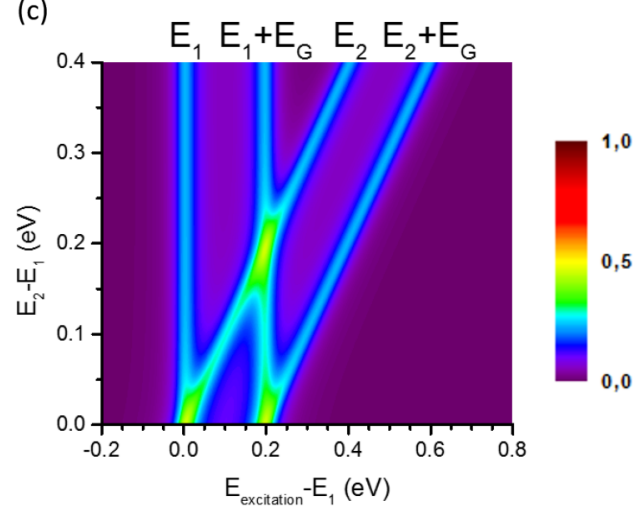

FIG. 1. Calculated dependence of the intensity of the LO $G$ mode and TO $G$ mode as a function of the incident energy and for different values of the gap between two consecutive transitions $\left(E_{1}\right.$ and $\left.E_{2}\right)$. (a) and (b) with interferences effects, (c) without interferences effects. In these calculations $E_{G}=0.2 \mathrm{eV}, \Gamma_{1}=\Gamma_{2}=25 \mathrm{meV} ; M_{1} / M_{2}=-1$ for LO mode and $M_{1} / M_{2}=1$ for TO mode (see text).

stated that for the $\mathrm{LO} G$ mode, $\mathrm{M}_{1}$ and $\mathrm{M}_{2}$ have opposite sign and, for the TO $G$ mode, all the $M_{i}(i=1,2)$ values are positive [6]. $\Gamma_{1}$ and $\Gamma_{2}$ are kept at $25 \mathrm{meV}$ which is the average value of the damping parameter derived from absorption experiments performed on individual SWNTs [7]. Despite the rough assumptions used, the simulation captures the main consequences of the quantum interferences on the relative intensity of the LO and TO $G$ modes. When the energy spacing between two consecutive transitions is approximately equal to the $G$ mode energy $\left(E_{2}-E_{1} \approx 0.2 \mathrm{eV}\right)$, the interferences lead to an enhancement of the intensity of the LO mode and a concomitant vanishing of the TO mode for an excitation energy $E_{L}$ close to the $E_{2}$ transition. By contrast, when the two consecutive transitions are close, an enhancement of the TO mode intensity and the vanishing of the LO mode are predicted for an excitation energy $E_{L}$ close to $E_{1}$ and $E_{1}+E_{G}$ energies. These plots are in qualitative agreement with the data of Duque et al. regarding the dependence of the intensity of the TO and LO $G$ modes on the laser excitation energy [4]. An illustration of the dependence of the intensity of the LO (or TO) $G$ mode without interference [expression (1)] is displayed in Fig. 1(c). Obviously, when no quantum interference effects are taken into account, the LO mode and TO mode show the same dependence on the excitation energy. In other words, without interferences, the $\mathrm{TO} / \mathrm{LO}$ intensity ratio is essentially constant for each transition.

Double-walled carbon nanotubes (DWNTs) are made of two concentric and weakly van der Waals coupled singlewalled carbon nanotubes (SWNTs). DWNTs are the most ideal and fundamental systems to explore the mechanical and electronic couplings between concentric carbon layers. A DWNT is uniquely characterized by the chiral indices $\left(n_{i}, m_{i}\right)$ and $\left(n_{o}, m_{o}\right)$ of the constituent inner and outer SWNTs, respectively. Hereafter the structure of a DWNT is identified as: $\left(n_{i}, m_{i}\right) @\left(n_{o}, m_{o}\right)$. Because the inner and outer SWNTs can be either semiconducting (SC) or metallic (M), DWNTs display four different configurations: SC@SC, SC@M, M@SC, and $\mathrm{M} @ \mathrm{M}$, which possess distinct electronic properties. All the properties of DWNTs are related to the individual nature of the layers and their interactions. Especially, the wall-to-wall distance, which typically ranges from $0.3 \mathrm{~nm}$ to $0.38 \mathrm{~nm}$, can affect the behavior of DWNTs by changing the strength of the wall-to-wall interactions [8].

The collective in-phase and out-of-phase radial breathing modes of both layers, the so-called radial breathinglike modes(RBLMs), are observed in the low-frequency range of the Raman spectrum of individual DWNTs [8,9]. Despite the collective nature of these modes, the breathing oscillation of the outer (inner) nanotube is the main contribution at the in-phase (out-of-phase) RBLM, especially in DWNTs of small diameters [10]. In relation to the collective nature of these modes, it was demonstrated that both RBLMs can be resonantly excited if an electronic transition of either constituent nanotube matches the excitation energy [8,9]. However the in-phase (out-of-phase) RBLMs tends to be the dominant component of the doublet when the outer (inner) nanotube is in resonance [9].

Depending on the chiral or achiral nature of each constituent SWNT, one expects to observe 4 (chiral@chiral), 3 (chiral@achiral or achiral@chiral), or 2 (achiral@achiral) components in the high-frequency $G$ mode range of the Raman spectrum measured on an individual DWNT. However, it must be emphasized that some components can appear at close frequencies and thus cannot be experimentally resolved. Recent experiments on individual index-identified DWNTs have permitted us to identify the contributions of each layer at the $G$ band of a DWNT [11,12]. Two couples of TO and LO $G$ modes, associated to inner and outer chiral layers, respectively, have been identified. On the other hand, the dependence of the $G$-modes frequencies on the diameter and interlayer distance in SC@SC DWNTs was reported [12]. Especially, it was found that the $\mathrm{TO}\left(G^{-}\right.$component) and LO $\left(G^{+}\right.$component $)$frequencies of the semiconducting inner layer shift significantly with respect to the frequencies of the same modes in constituent SWNTs, the direction and values of the shift depending on the interlayer distance.

The optical properties of index-identified DWNTs have been recently investigated by spatial modulation spectroscopy technique [13], absorption spectroscopy [14], and Rayleigh spectroscopy [15]. A one-to-one correspondence between the DWNT optical resonances, hereafter named $S_{i i \text {,out }}\left(M_{i i, \text { out }}\right)$ for the semiconducting (metallic) outer tube and $S_{i i \text {,in }}\left(M_{i i \text {,in }}\right)$ for 
the semiconducting (metallic) inner tube, and those from the constituent SWNTs was found. However, the optical transition energy shift varies in a large energy range: from a redshift of about $200 \mathrm{meV}$ to a blueshift of about $50 \mathrm{meV}$ in different DWNTs species [14]. It must be emphasized that the excitation dependence of the Raman-active modes (RBLM and $G$ modes) combined with absorption measurements performed on the same individual DWNTs is an efficient tool to unambiguously assign the origin of the different optical transitions [11,13].

Here, we report optical absorption and resonant Raman scattering experiments on two individual free-standing DWNTs index identified by electron diffraction. The Raman spectra of the $(16,12) @(27,10)$ DWNT show a total vanishing of the TO mode of the outer tube for a $1.55 \mathrm{eV}$ laser excitation. By contrast, the Raman spectra excited around $2.5 \mathrm{eV}$ reveal an enhancement of the intensity of TO mode of the outer tube mode concomitant with the decreasing of the intensity of the LO $G$ mode of the outer tube. Both distinct behaviors are well understood in terms of interference effects. On the other hand, in the $(15,9) @(22,12)$ DWNT, the distortion of the REP of the $\mathrm{LO} G$ mode of the metallic inner tube $\left(\mathrm{LO}_{\text {in }}\right)$ measured in the $1.45-1.7 \mathrm{eV}$ excitation range is explained in terms of interference due to the proximity of the low $\left(M_{11-\text {,in }}\right)$ and high $\left(M_{11+\text {,in }}\right)$ components of the $M_{11 \text {,in }}$ transition of the metallic inner tube.

The experimental information is described in Sec. II. The results are presented and discussed in Sec. III.

\section{SAMPLE AND EXPERIMENTAL METHODS}

Ultralong individual double-walled nanotubes were grown by catalytic chemical vapor deposition (CCVD) method directly on homemade slits fabricated by wet-etching of a $\mathrm{Si}_{x} \mathrm{~N}_{y} / \mathrm{Si}$ wafer (for details, see Ref. [16]). High-resolution transmission electronic microscopy (HRTEM) images and electron diffraction (ED) patterns were recorded in a FEI Titan microscope operating at $80 \mathrm{kV}$ and within short acquisition times (less than $5 \mathrm{~s}$ for $\mathrm{ED}$ ) to reduce damage induced by electron diffraction $[17,18]$. Direct measurements of the absolute absorption cross-section spectra of freely suspended individual DWNTs were assessed via spatial modulation spectroscopy technique (for details, see Refs. [7,19]). The resonant Raman scattering measurements were performed on a homemade setup, including an iHR-550 Horiba spectrometer equipped with a liquid-nitrogen-cooled silicon CCD detector, with a wide variety of laser excitation energies, covering the 1.44-2.71 eV range, by using $\mathrm{Ar}^{+}, \mathrm{Kr}^{+}, \mathrm{He}-\mathrm{Ne}$ lasers and tunable Ti:sapphire and Dye lasers filtered using tunable laser line filters. The scattered light was collected through a $100 \times$ objective (N.A. $=0.95$ ) using a back-scattering configuration. The laser power impinging the sample was kept below $100 \mu \mathrm{W}$. In these conditions, with a laser spot full width at half maximum about $500 \mathrm{~nm}$, heating effects are avoided. In all the measurements, both incident and scattered light polarizations are along the nanotube axis. To obtain the resonance excitation profile (REP), the experimental intensity of each mode measured at a specific excitation energy was normalized by the intensity of the $521 \mathrm{~cm}^{-1}$ line of silicon [Si (111)] which was taken as a reference sample and corrected by the calibrated total transmission of our optical system for the different scattered light polarizations to take into account the difference in sensitivity at the absolute wavelength of the measured nanotubes modes and at the absolute wavelength of the Si line [20].

\section{RESULTS AND DISCUSSION}

An individual free-standing DWNT, suspended across a trench [see the HRTEM image of this tube in Fig. 2(a), inset], was unambiguously index identified as $(16,12) @(27,10)$ (SC@SC) by combining electronic diffraction [Fig. 2(a)], the frequencies of the radial breathinglike mode (RBLMs) [Figs. 3(a) and 3(c)], and the line shape and frequencies of the $G$ modes [Figs. 3(b) and 3(d)]. The method of identification combining ED and Raman information is presented in detail in Refs. [11,18].

The absorption spectrum of the $(16,12) @(27,10)$ DWNT (1.905 nm@2.595 nm), measured in the 1.3-3 eV range, is displayed in Fig. 2(b). Combining the absorption data with the excitation dependence of the in-phase RBLM, located at $109 \mathrm{~cm}^{-1}$, and of the out-of-phase RBLM, located at $142 \mathrm{~cm}^{-1}$ [Figs. 3(a) and 3(c)] permits us to unambiguously assign
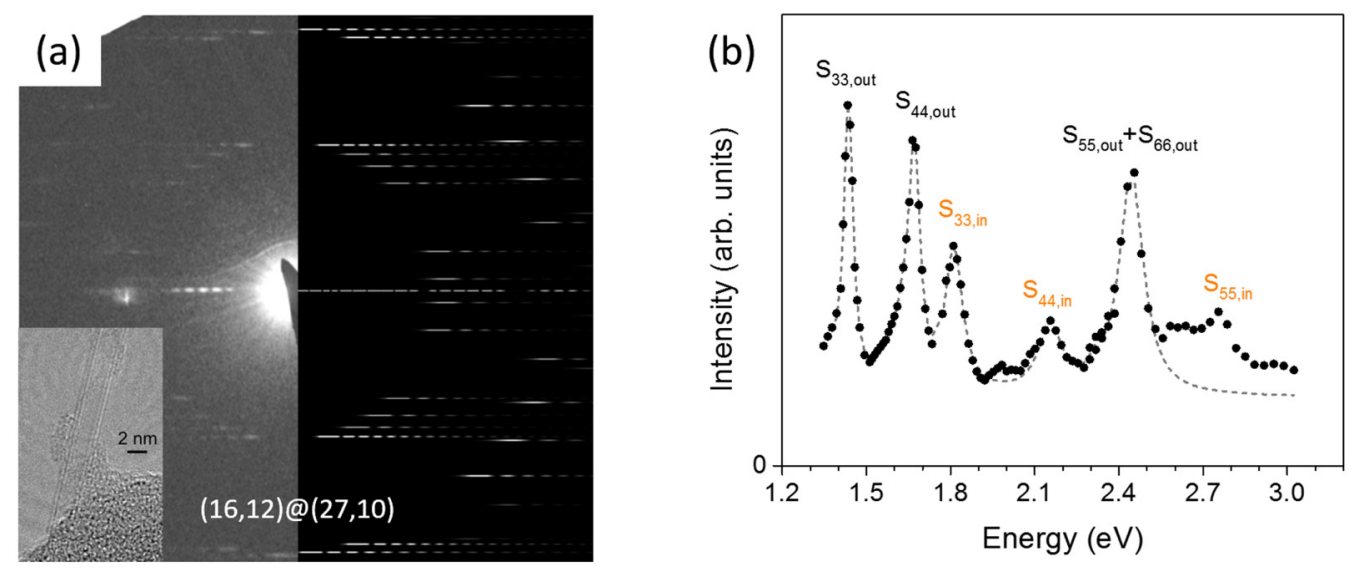

FIG. 2. (a) Experimental (left) and simulated (right) electron diffraction patterns of the $(16,12) @(27,10)$ DWNT. Inset: HRTEM micrograph of the DWNT. (b) Optical absorption spectrum of the individual $(16,12) @(27,10)$ DWNT, experimental data (dots) and fit (dotted line). The assignment of the optical transitions are labeled as described in the main text in the $1.2-3 \mathrm{eV}$ range. 
(a)

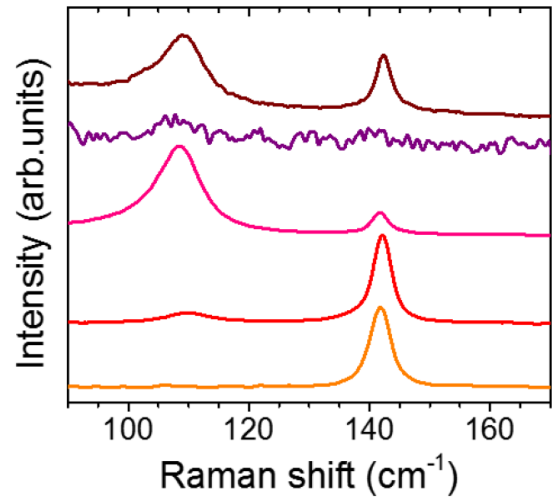

(c)

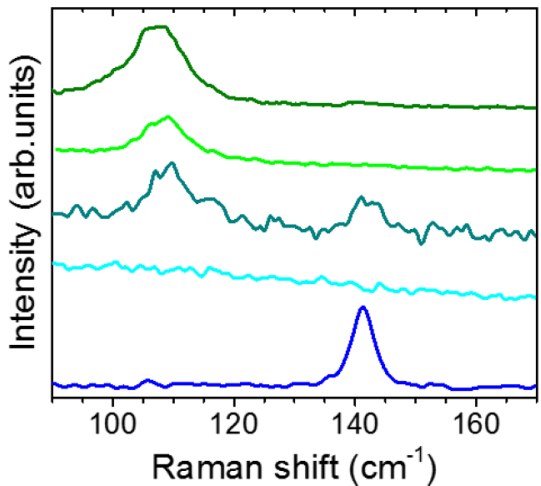

(b)

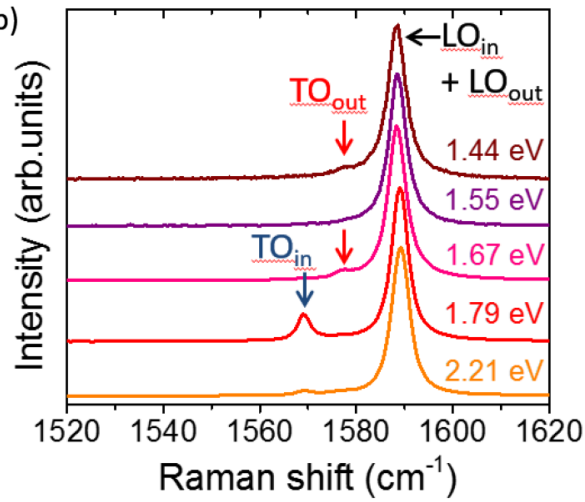

(d)

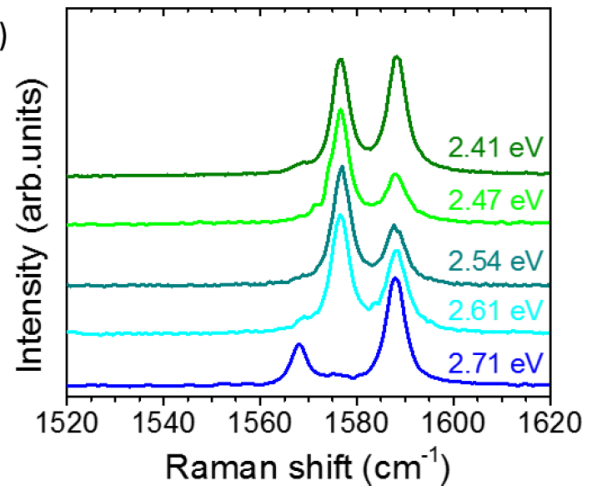

FIG. 3. Raman spectra of the (16,12)@(27,10) excited at (a), (b) 1.44, 1.55, 1.67, 1.79, and $2.21 \mathrm{eV}$, and (c), (d) 2.41, 2.47, 2.54, 2.61, and $2.71 \mathrm{eV}$ as labeled on the graphs. (a)-(c) RBLM range, (b)-(d) $G$ mode range. In (b), the red, blue, and black arrows highlight the positions of the $\mathrm{TO}_{\text {out }}, \mathrm{TO}_{\text {in }}$, and $\mathrm{LO}_{\text {in }}+\mathrm{LO}_{\text {out }}$, respectively.

these transitions as originating from the outer and inner tubes, respectively. Absorption spectrum displays well defined peaks at 1.44, 1.67, 1.81, 2.15, 2.44, and $2.75 \mathrm{eV}$ [Fig. 2(b)]. Because we observe a strong intensity for the in-phase RBLMs at excitation energies close to 1.44 and $1.67 \mathrm{eV}$ [Fig. 3(a)], we assign these transitions as originating from the semiconducting outer tubes. The diameter of the outer tube being $2.595 \mathrm{~nm}$, we infer that the transitions at 1.44 and $1.67 \mathrm{eV}$ are the $S_{33 \text {,out }}$ and $S_{44, \text { out }}$ transitions, respectively [21]. Due to the strong resonance of the in-phase RBLM at excitation energy close to $2.44 \mathrm{eV}$ [Fig. 3(c)], the absorption peak at $2.44 \mathrm{eV}$ is also associated with optical transition of the semiconducting outer tube. In the Kataura plot established for SWNTs [21], which can be applied to DWNTs to a first approximation, the $E_{55}$ and $E_{66}$ are very close suggesting that the broad absorption peak at $2.44 \mathrm{eV}$ is a mix of $S_{55, \text { out }}$ and $S_{66 \text {,out }}$ transitions [21]. The peaks at $1.81,2.15$, and $2.71 \mathrm{eV}$ are associated with optical transitions of the semiconducting inner tube since the out-of-phase RBLM dominates the Raman spectra recorded close to these energies [Figs. 3(a) and 3(c)]. With regards to the diameter of the inner tube $(1.905 \mathrm{~nm})$, these absorption energies are assigned to the $S_{33 \text {,in }}, S_{44 \text {,in }}$, and $S_{55 \text {,in }}$ transitions, respectively [21]. On the basis of the attribution of the optical transitions, we first analyze the excitation dependence of the RBLM and $G$ modes in the 1.3-2.1 eV energy range where the $S_{33 \text {,out }}, S_{44 \text {,out }}$ and $S_{33 \text {,in }}$ transitions are located.

Figure 3(a) displays the Raman spectra in the RBLM range measured at five excitation energies, namely: 1.44, 1.55, 1.67, 1.79 , and $2.21 \mathrm{eV}$. In order to detail the excitation dependence of both RBLMs, the resonance excitation profile (REP) of the in-phase RBLM [Fig. 4(a)] and the one of the out-of-phase RBLM [Fig. 4(b)] were measured. First, these REPs provide a clear illustration that RBLMs can be resonantly excited if an electronic transition of either wall matches the excitation energy. For instance, both in-phase and out-of-phase RBLMs show resonances at 1.44, 1.67, and $1.81 \mathrm{eV}$ corresponding to the $S_{33 \text {,out }}, S_{44 \text {,out }}$, and $S_{33 \text {,in }}$ transitions, respectively. However, it must be emphasized when a resonance condition of the outer (inner) tube is achieved, the RBLM intensity of the in-phase (out-of-phase) RBLM is always stronger than that of the out-of phase (in-phase) RBLM. As expected, both fits of the REPs, with and without interference effects, well reproduce the experimental data and give the same values of the resonance energies $\left(S_{33 \text {, out }}=1.44 \mathrm{eV}, S_{44, \text { out }}=1.67 \mathrm{eV}\right.$, and $\left.S_{33 \text {,in }}=1.81 \mathrm{eV}\right)$ and close damping parameters $\left(\gamma_{33 \text {,out }}=\right.$ $20 \mathrm{meV}, \gamma_{44, \text { out }}=24 \mathrm{meV}$, and $\gamma_{33 \text {,in }}=33 \mathrm{meV}$ ) to the ones derived from the absorption spectrum $\left(\gamma_{33 \text {,out }}=20 \mathrm{meV}\right.$, $\gamma_{44, \text { out }}=25 \mathrm{meV}$, and $\gamma_{33 \text {,in }}=30 \mathrm{meV}$ ).

Figure 3(b) compares the profiles of the Raman spectra in the $G$ modes range measured at $1.44,1.55,1.67,1.79$, and $2.21 \mathrm{eV}$. At the resonance with the $S_{33 \text {,out }}$ transition $(1.44 \mathrm{eV})$, two components located at $1589 \mathrm{~cm}^{-1}$ (strong) and $1577 \mathrm{~cm}^{-1}$ (weak) are observed and assigned to the LO and TO modes of the chiral $(27,10)$ outer tube (hereafter these modes are called $\mathrm{LO}_{\text {out }}$ and $\mathrm{TO}_{\text {out }}$ respectively). On the other hand, the spectrum excited at $1.79 \mathrm{eV}$, close to the $S_{33 \text {,in }}$ transition (1.81 eV), and the one excited at $2.21 \mathrm{eV}$, close to the $S_{44 \text {,in }}$ transition $(2.15 \mathrm{eV})$, display two dominant contributions at $1589 \mathrm{~cm}^{-1}$ (strong) and $1568 \mathrm{~cm}^{-1}$ (weak) assigned to the LO and TO modes of the chiral $(16,12)$ inner tube (hereafter these 
(a)

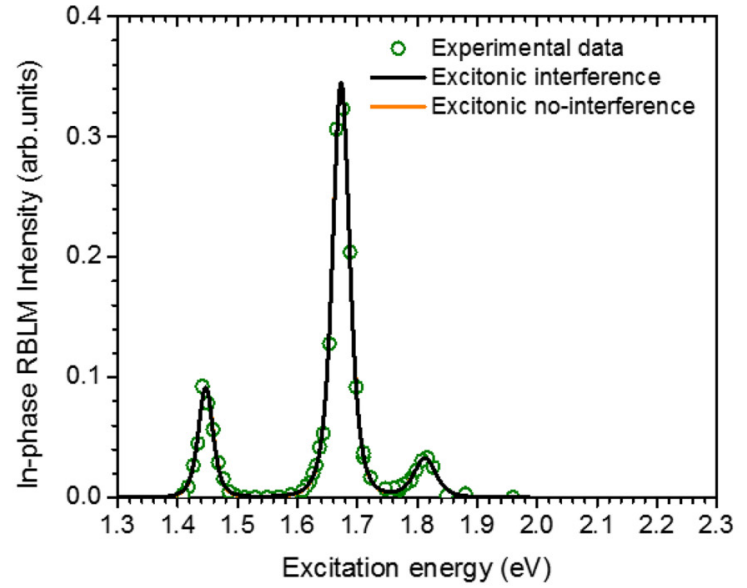

(c)

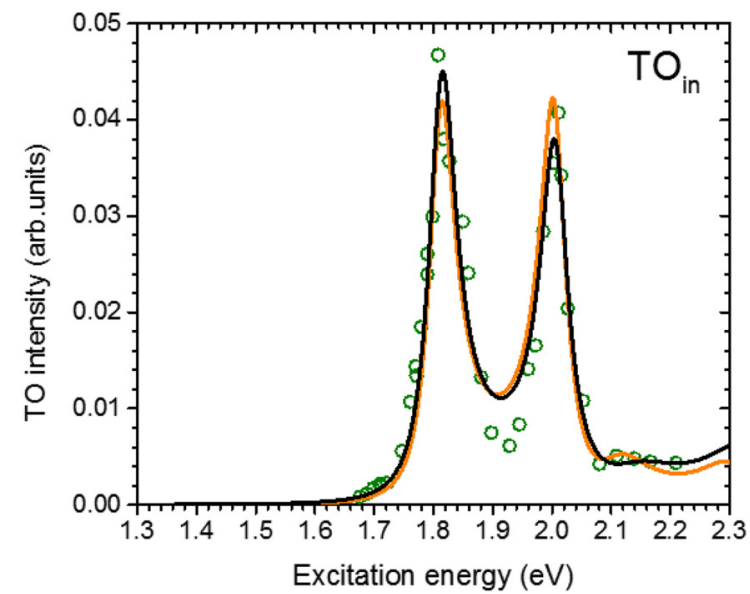

(b)

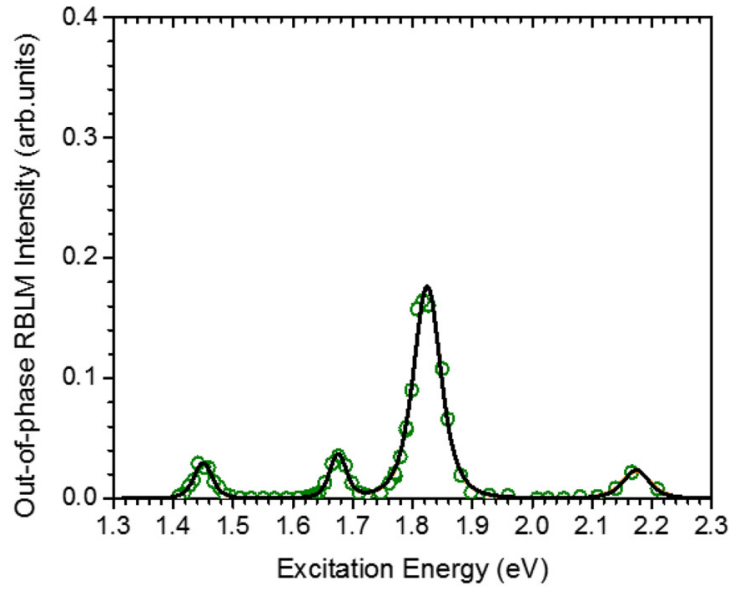

(d)

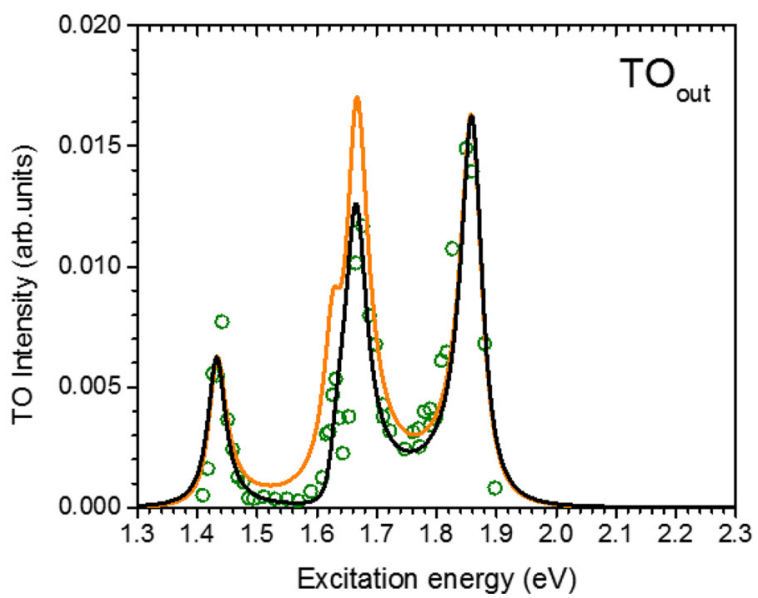

FIG. 4. (a) REP of the in-phase RBLM, (b) REP of the out-of-phase RBLM, (c) REP of the TO in $_{\text {mode, (d) REP of the TO }}$ out mode. Green open dots, experimental data. Black (orange) solid line, fits or simulations with (without) interference effects (see main text).

modes are called $\mathrm{LO}_{\text {in }}$ and $\mathrm{TO}_{\text {in }}$, respectively). In agreement with previous results established in chiral semiconducting SWNTs [22,23], the TO components are weak but well defined in the spectra. It must be emphasized that the excitation dependence of the Raman spectra unambiguously states an overlap of $\mathrm{LO}_{\text {in }}$ and $\mathrm{LO}_{\text {out }}$ modes around $1589 \mathrm{~cm}^{-1}$. Striking, in the spectrum excited at $1.55 \mathrm{eV}$, the $\mathrm{TO}_{\text {out }}$ mode totally vanishes. We attribute this change in the profile of the $G$ mode to an effect of quantum interferences. In order to investigate in detail the excitation dependence of the well separated $\mathrm{TO}_{\text {in }}$ and $\mathrm{TO}_{\text {out }}$ modes, the REP of each mode was measured in the 1.3-2.1 eV excitation range [Figs. 4(c) and 4(d), respectively).

For $\mathrm{TO}_{\text {in }}$, two intensity maxima located at $1.81 \mathrm{eV}$ and close to $2 \mathrm{eV}$ are observed and assigned to $S_{33 \text {,in }}$ incident and outgoing resonance processes. As expected, since the $S_{33 \text {,in }}$ and $S_{44 \text {,in }}$ are separated by more than $0.3 \mathrm{eV}$, the fits of the REPs with and without interference effects well reproduce the experimental data. Furthermore, these fits give the same value for the transition energy $\left(S_{33 \text {,in }}=1.81 \mathrm{eV}\right)$ and a damping parameter $\left(\Gamma_{33 \text {,in }}=29 \mathrm{meV}\right)$ close to the one derived from the analysis of the absorption spectrum $\left(\Gamma_{33 \text {, in }}=30 \mathrm{meV}\right)$ [Fig. 4(c)]. It is worth noting that such an agreement on the values of the excitonic transition energy and damping parameter derived from the analysis of the REP and the optical absorption spectrum has been recently reported for SWNTs [20].

Because two transitions of the outer tube are measured in the $1.3-2.1 \mathrm{eV}$ energy range, namely $S_{33 \text {,out }}$ and $S_{44 \text {,out }}$, four peaks are expected in the REP of the $\mathrm{TO}_{\text {out }}$ mode [Fig. 4(d)] corresponding to the $S_{33 \text {,out }}, S_{33 \text {,out }}+E_{G}, S_{44 \text {,out }}$, and $S_{44, \text { out }}+E_{G}$ energies, respectively. Due to the proximity of the $S_{33 \text {,out }}+E_{G}$ and $S_{44 \text {,out }}$ energies, quantum interference effects are predicted to occur in this range (see Fig. 1). To put in evidence of these effects we compare the simulation of the REP of the $\mathrm{TO}_{\text {out }}$ mode with and without interference effects. In these simulations, the energies and damping parameters of the optical transitions are fixed to their values derived from the absorption spectrum. The energies of the $S_{33 \text {,out }}$ and $S_{44, \text { out }}$ transitions are fixed at 1.44 and $1.67 \mathrm{eV}$, respectively and the damping parameters are $20 \mathrm{meV}$ for the $S_{33 \text {,out }}$ transition and $25 \mathrm{meV}$ for the $S_{44, \text { out }}$ transition [13]. The value of $M_{1}$ and $M_{2}$ are adjusted from the intensity of the peaks around $1.44 \mathrm{eV}$ and $1.88 \mathrm{eV}$, respectively, because interference effects can be neglected in these respective energy ranges. Figure 4(d) compares the results of the simulation with [Fig. 4(d), black solid line] and without [Fig. 4(d)], orange solid line) interference effects. Unambiguously, the simulation taking into account interference effects well reproduces the 
(a)

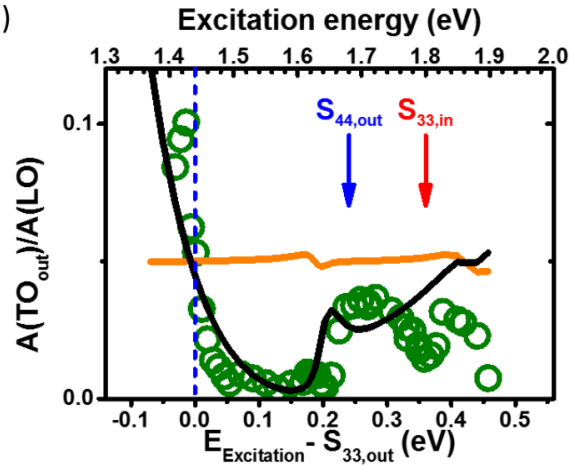

(c)

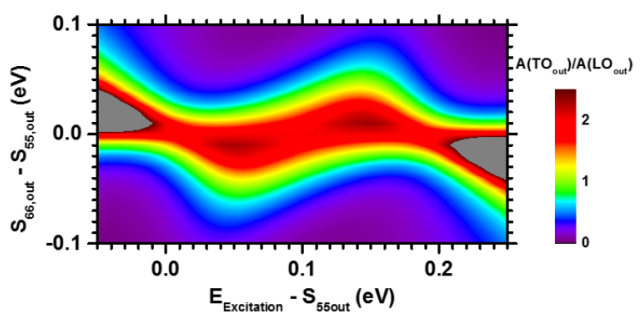

(b)

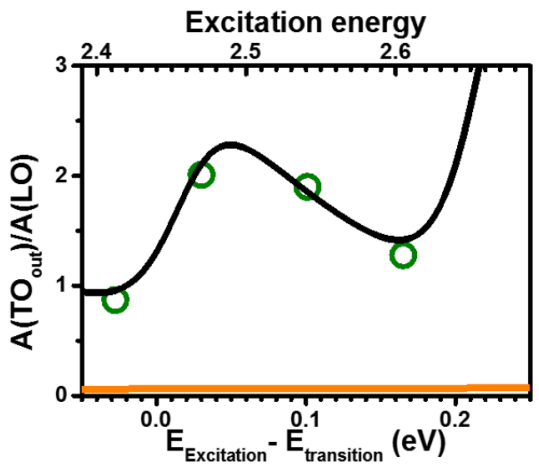

(d)

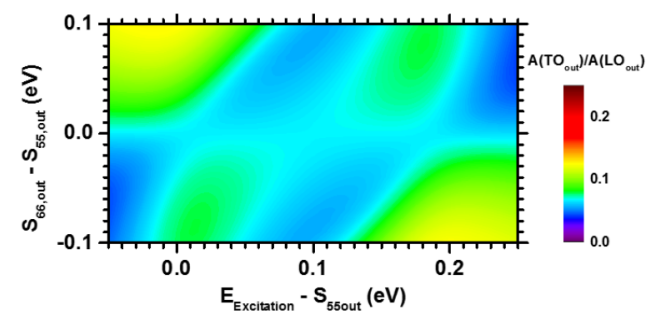

FIG. 5. (a) $\left(\mathrm{TO}_{\text {out }}\right.$ mode) $/\left(1589 \mathrm{~cm}^{-1}\right.$ component) integrated intensity ratio as a function of excitation energy in the $1.4-1.9 \mathrm{eV}$ (top $x$ axis), and relative to the $S_{33 \text {,in }}$ transition at $1.44 \mathrm{eV}$ marked by the vertical blue dashed line (bottom $x$ axis). Green open dots, experimental data. Black (orange) solid line, simulations with (without) interference effects. The blue (resp. red) arrow points the $S_{44, \text { out }}$ (resp. $S_{33 \text {,in }}$ ) transition energy. (b) $\mathrm{TO}_{\text {out }}$ mode $/\left(1589 \mathrm{~cm}^{-1}\right.$ component) intensity ratio as a function of the excitation energy in the $2.4-2.6 \mathrm{eV}$ range (top $x$ axis), and relative to the $2.44 \mathrm{eV}$ transition $S_{55 \text {,out }}+S_{66, \text { out }}$ (bottom $x$ axis). Green open dots, experimental data. Black (orange) solid line, simulations with (without) interference effects for $S_{66, \text { out }}-S_{55, \text { out }}=-10 \mathrm{meV}$ extracted from (c) and (d) maps, respectively. (c), (d) Maps of simulations of the $\mathrm{TO}_{\text {out }} / L O_{\text {out }}$ intensity ratio as a function of the $S_{55 \text {,out }}$ and $S_{66 \text {,out }}$ energy separation and of the relative excitation energy with (c) and without (d) interference effect. In the simulations, $M_{1}(\mathrm{TO})=1, M_{2}(\mathrm{TO})=0.65, M_{1}(\mathrm{LO})=2.65$, and $M_{2}(\mathrm{LO})=-3.82$, and the damping parameter of both transitions is fixed to the value extracted from the absorption spectrum, i.e., $48 \mathrm{meV}$.

experimental $\mathrm{REP}$ of the $\mathrm{TO}_{\text {out }}$ mode in all the investigated excitation energy range. As expected, the interference effects lead to a general decreasing of the intensity of the $\mathrm{TO}_{\text {out }}$ mode in the $1.5-1.75 \mathrm{eV}$ range where the $S_{33 \text {,out }}+E_{G}$ and $S_{44, \text { out }}$ transition are close and a complete vanishing of the intensity of the $\mathrm{TO}_{\text {out }}$ mode around $1.55 \mathrm{eV}$ as observed experimentally.

Another illustration of the interference effects is given by the comparison of the experimental $\mathrm{TO}_{\text {out }} \operatorname{mode} /\left(1589 \mathrm{~cm}^{-1}\right.$ component) integrated intensity ratio with the simulation in the 1.4-1.9 eV energy range [Fig. 5(a)]. The simulation of this ratio by taking into account interference effects [Fig. 5(a), black solid line) well reproduces the experimental behavior (Fig. 5(a), green open dots) in the $1.4-1.75 \mathrm{eV}$ range in which the signal at $1589 \mathrm{~cm}^{-1}$ is mainly assigned to the $\mathrm{LO}_{\text {out }}$ mode. Above $1.75 \mathrm{eV}$, due to the presence of the $S_{33 \text {,in }}$ transition at $1.81 \mathrm{eV}$, the $\mathrm{LO}_{\text {in }}$ mode contributes to the $1589 \mathrm{~cm}^{-1}$ peak intensity leading to a decrease of the ratio. It must be emphasized that the simulation of this ratio without interference effects leads to a flat dependence of this ratio in complete disagreement with the experimental data [Fig. 5(a), orange solid line]. This evidences the crucial role of interferences especially regarding the vanishing of the $\mathrm{TO}_{\text {out }}$ mode around $1.55 \mathrm{eV}$. It can be emphasized that the profile of the Raman spectrum excited at $1.55 \mathrm{eV}$ alone could lead to the wrong conclusion that a semiconducting zigzag tube is present in this DWNT [24].

In the following, we analyze the excitation dependence of the $G$ modes in the $2.41-2.71 \mathrm{eV}$ excitation range (Fig. 3(d)) where are located the $S_{55 \text {,out }}$ and $S_{66, \text { out }}$ transitions. For excitations in the 2.41-2.61 eV range, the components located at $1589 \mathrm{~cm}^{-1}$, assigned to a mix of $\mathrm{LO}_{\text {out }}$ and $\mathrm{LO}_{\text {in }}$ modes, and $1577 \mathrm{~cm}^{-1}$, assigned to the $\mathrm{TO}_{\text {out }}$ mode, dominate the spectra [Fig. 3(d)]. Striking is the intensity of the $\mathrm{TO}_{\text {out }}$ mode which is significantly stronger that the one of the $1589 \mathrm{~cm}^{-1}$ component. This excitation range matches the transition at $2.44 \mathrm{eV}$ assigned to a mix of $S_{55 \text {, out }}$ and $S_{66, \text { out }}$ transition of the $(27,10)$ outer tube. As expected from the simulations of the LO and TO intensity (see Fig. 1), for two close transitions, constructive (destructive) quantum interference effects occur for TO (LO) $G$ mode. The relative strong intensity of the $\mathrm{TO}_{\text {out }}$ mode is in qualitative agreement with the results of the simulations.

The excitation energy dependence of the $\mathrm{TO}_{\text {out }}$ mode $/\left(1589 \mathrm{~cm}^{-1}\right.$ component $)$ integrated intensity ratio is shown on Fig. 5(b) (green dots) in the 2.4-2.6 excitation range. In this energy range, where no transition of the inner tube occurs, the contribution of the $\mathrm{LO}_{\text {in }}$ mode at the $1589 \mathrm{~cm}^{-1}$ component can be neglected in the first approximation. This ratio displays a broad profile centered around $2.5 \mathrm{eV}$. We performed simulations of the $\mathrm{TO}_{\text {out }} / \mathrm{LO}_{\text {out }}$ ratio excitation profile as a function of the energy difference between $S_{55 \text {,out }}$ and $S_{66, \text { out }}$ taking into account interferences effects [Fig. 5(c)] or not [Fig. 5(d)]. As shown in Fig. 5(b), a quantitative agreement can be found between experimental data [Fig. 5(b)], green open dots) and simulations with interferences for an $S_{55, \text { out }}$ energy of $10 \mathrm{meV}$ higher than the $S_{66, \text { out }}$ one [Fig. 5(b), 

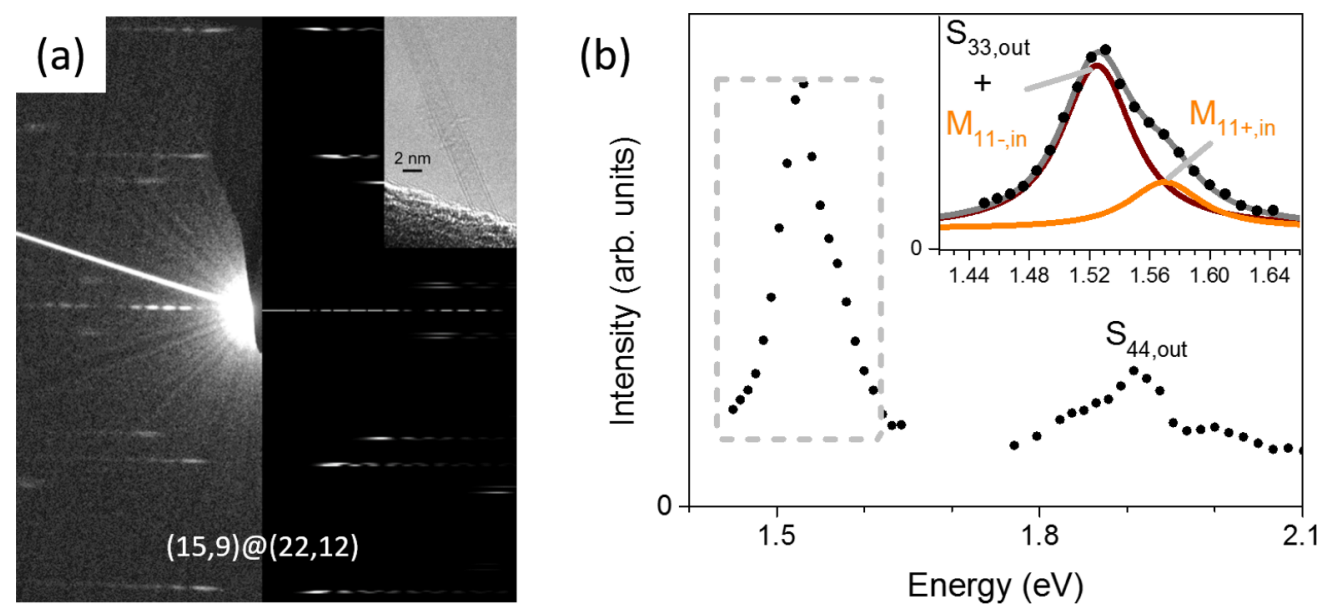

FIG. 6. (a) Experimental (left) and simulated (right) electron diffraction patterns of the $(15,9) @(22,12)$ DWNT. Inset: HRTEM micrograph of the DWNT. (b) Optical absorption spectrum of the individual $(15,9) @(22,12)$ DWNT in the $1.4-2.1 \mathrm{eV}$ range. Inset: zoom on the lowest energy optical absorption band. Black filled dots experimental data and full lines fit. The assignment of the optical transitions are labeled as described in the main text.

black solid line]. This value of the separation in energy of the transition should be taken as an estimate since it depends slightly on the parameters used for the simulation. Nevertheless, the important point here is that the simulations without interferences effects lead in all cases [Fig. 5(d)] to a ratio more than one order of magnitude lower than the experimental one and to flat excitation energy dependence of this ratio in complete disagreement with the experimental data [Fig. 5(b), orange solid line].

Finally, it must be emphasized that the excitation dependence of each $G$ mode in DWNT can be an efficient help to unambiguously assign each of them as originating from the LO or TO $G$ modes of inner and outer tubes. Here, for instance, the peculiar excitation dependence of the $1577 \mathrm{~cm}^{-1}$ mode permits us to unambiguously identify this one as the $\mathrm{TO}_{\text {out }}$ mode and not as the $\mathrm{LO}_{\text {in }}$ mode.

Another individual free-standing DWNT [see its HRTEM image in the inset of Fig. 6(a)] has been unambiguously index identified as $(15,9) @(22,12)(\mathrm{M} @ S C)(1.644 @ 2.34)$ by combining the information provided by electronic diffraction [Fig. 6(a)], the frequencies of the radial breathinglike mode (RBLMs) [Fig. 7(a)], and the line shape and frequencies of (a)

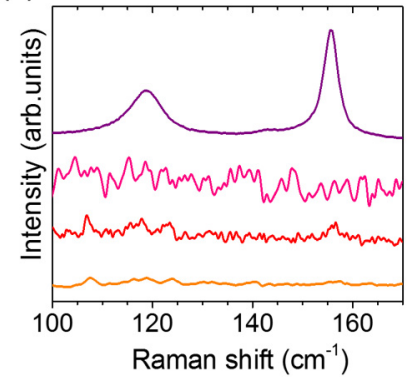

(b) G modes

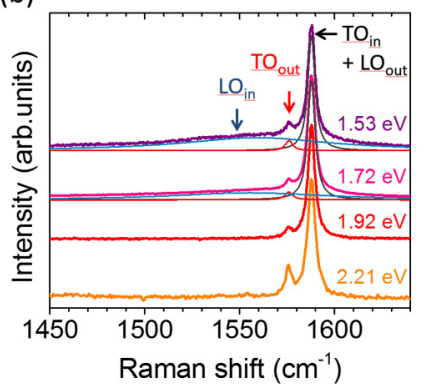

FIG. 7. Raman spectra of the $(15,9) @(22,12)$ DWNT excited at $1.53,1.72,1.92$, and $2.21 \mathrm{eV}$ as labeled on the graph. (a) RBLM range, (b) $G$ mode range. In (b), the red, blue, and black arrows highlight the positions of the $\mathrm{TO}_{\text {out }}, \mathrm{LO}_{\text {in }}$ and $\mathrm{TO}_{\text {in }}+\mathrm{LO}_{\text {out }}$, respectively.
$G$ modes [Fig. 7(b)] [11,18]. Its absorption spectrum, measured in the 1.4-2.1 eV range [Fig. 6(b)], show two bands located around $1.55 \mathrm{eV}$ and $1.91 \mathrm{eV}$. However a detailed analysis of the profile of the first band [Fig. 6(b), inset] permits us to identify two close transitions, namely a strong one at $1.53 \mathrm{eV}$ [Fig. 6(b), inset, magenta solid line] and a weak one at $1.57 \mathrm{eV}$ [Fig. 6(b), inset, orange solid line].

The RBLM and $G$ mode ranges of the Raman spectra excited at 1.53, 1.72, and $1.92 \mathrm{eV}$ are displayed in Figs. 7(a) and 7(b), respectively. At the $1.92 \mathrm{eV}$ incident excitation, no RBLM is observed [Fig. 7(a)] and two narrow and symmetric components are measured in the $G$ modes range at $1588 \mathrm{~cm}^{-1}$ and $1576 \mathrm{~cm}^{-1}$ [Fig. 7(b)]. At $1.53 \mathrm{eV}$ and $1.72 \mathrm{eV}$ excitation energies, an additional weak and broad component centered around $1558 \mathrm{~cm}^{-1}$ is measured [Fig. 7(b)]. At $1.53 \mathrm{eV}$, the in-phase RBLM, located at $120 \mathrm{~cm}^{-1}$, and the out-of-phase RBLM, located at $150 \mathrm{~cm}^{-1}$, are observed, and both RBLMs vanish in the spectrum excited at $1.72 \mathrm{eV}$ [Fig. 7(a)].

By combining absorption [Fig. 6(b)] and Raman information [Figs. 7(a) and 7(b)] the following conclusions are achieved. The absorption peak at $1.91 \mathrm{eV}$ is assigned to $S_{44 \text {,out }}$. Three arguments support this assignment: (i) the narrow and symmetric profile of the $1588 \mathrm{~cm}^{-1}$ and $1576 \mathrm{~cm}^{-1} G$ modes measured at the $1.92 \mathrm{eV}$ excitation identifies a semiconducting tube and, consequently, these lines are assigned to $\mathrm{LO}_{\text {out }}$ and $\mathrm{TO}_{\text {out }} G$ modes, respectively. (ii) The nonobservation of RBLMs for $1.92 \mathrm{eV}$ excitation is in agreement with calculations which predict a zero amplitude of the RBM of the $(22,12)$ tube in the Raman spectrum excited around its $S_{44}$ transition [6]. (iii) The same profile of the $G$ modes in the Raman spectrum excited at $2.21 \mathrm{eV}$ than in the one excited at $1.92 \mathrm{eV}$ [Fig. 7(b)] is understood in terms of an outgoing resonance process in relation to an optical transition of the semiconducting outer tube close to $2 \mathrm{eV}$.

In the Raman spectrum excited at $1.53 \mathrm{eV}$, the observation of the in-phase and out-of-phase RBLM, with close intensities, and that of three $G$ modes indicate that both the metallic inner tube, identified from the presence of the broad component at $1588 \mathrm{~cm}^{-1}$ assigned to the $\mathrm{LO}_{\text {in }}$ mode, and semiconducting 
(a)

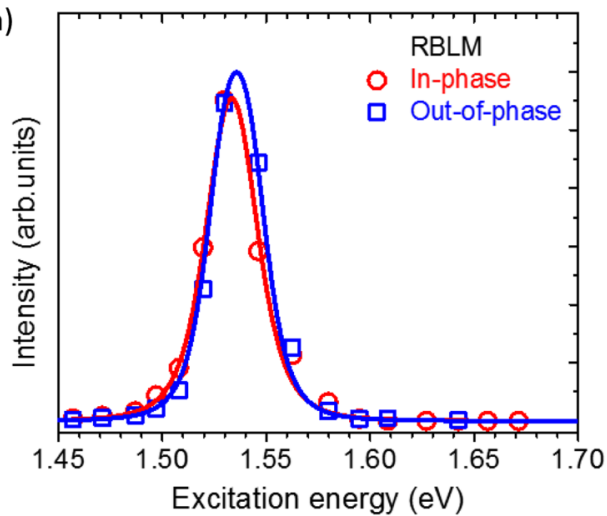

(c)

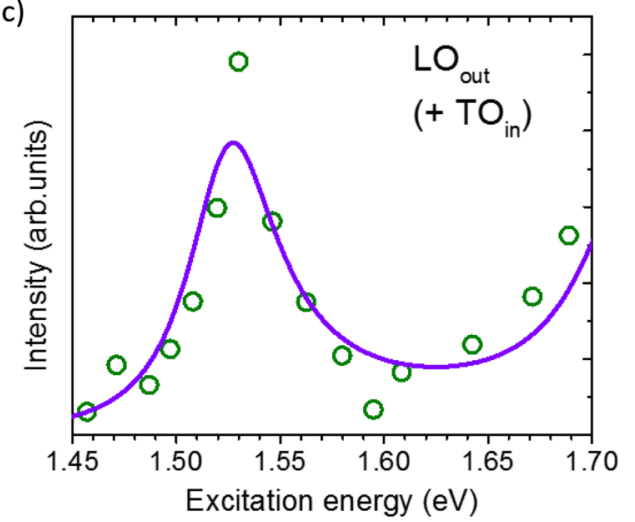

(b)

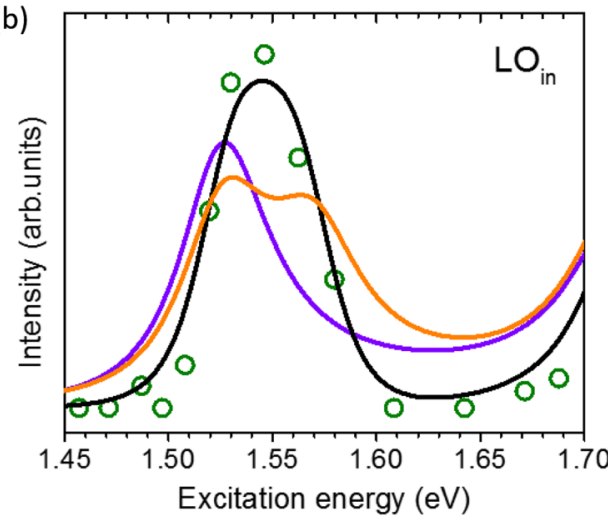

(d)

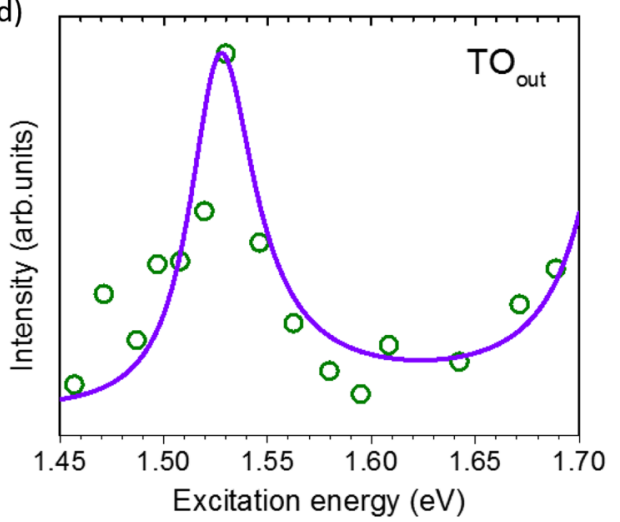

FIG. 8. REPs of the $(15,9) @(22,12)$ DWNT in the $1.45-1.70 \mathrm{eV}$ range. (a) REP of the in-phase RBLM (red open dots) and out-of-phase RBLM (blue open squares); red (blue) lines are the results of the fit by considering a single transition located at $1.53 \mathrm{eV}$ (see text). (b) Experimental (green open dots) and fit (solid lines) of the REP of $\mathrm{LO}_{\text {in }}$ mode. The solid lines correspond to the fit of the REP with (black line) and without (orange line) interferences between the 1.53 and $1.57 \mathrm{eV}$ transitions; violet solid line is the result of the fit by considering a single transition at $1.53 \mathrm{eV}$. (c) Experimental (green open dots) and fit (violet solid line) of the REPs of the $1589 \mathrm{~cm}^{-1} \mathrm{component}^{(m i x}$ of $\mathrm{TO}_{\text {in }}$ and $\mathrm{LO}_{\text {out }}$ ) by considering a single transition at $1.53 \mathrm{eV}$. (d) Experimental (green open dots) and fit (violet solid line) of the REP of TO by considering a single transition at $1.53 \mathrm{eV}$.

outer tube, identified from the presence of narrow components at $1588 \mathrm{~cm}^{-1}$ and $1576 \mathrm{~cm}^{-1}$, are in resonance at this excitation energy (note that this analysis suggests that the $\mathrm{TO}_{\text {in }}$ mode of the metallic inner tube and the $\mathrm{LO}_{\text {out }}$ mode of the semiconducting outer tube overlap around $\left.1588 \mathrm{~cm}^{-1}\right)$. From the diameter of inner $(1.644 \mathrm{~nm})$ and outer $(2.34 \mathrm{~nm})$ tubes, and in reference to the optical transitions in the corresponding SWNTs, we infer that the absorption band at $1.53 \mathrm{eV}$ is a mix of the $M_{11 \text {,in }}$ transition of the metallic inner tube and of the $S_{33 \text {,out }}$ transition of the semiconducting outer tube. This is consistent with the large absorption intensity measured at $1.53 \mathrm{eV}$. In addition for a metallic SWNT (except armchair), due to trigonal warping, each transition $M_{i i}$ splits in two components, one at a high energy, the so-called $M_{i i+}$ transition, the other one at low energy, the so-called $M_{i i-}$ transition. As shown in the inset of Fig. 6(b), the presence of a weak transition at $1.57 \mathrm{eV}$ permits us to state that the $M_{11-\text {,in }}$ transition contributes to the absorption at $1.53 \mathrm{eV}$ and the absorption peak at $1.57 \mathrm{eV}$ is assigned to the $M_{11+\text {,in }}$ transition. Our attribution compares well with the one measured on the $(15,9) @(25,8)$ DWNT in which the $M_{11+\text {,in }}$ and $M_{11+\text {,in }}$ transitions of the $(15,9)$ metallic inner tube were reported at $1.49 \mathrm{eV}$ and $1.61 \mathrm{eV}$, respectively [14]. Finally, the same profile of the $G$ modes in the spectrum excited at $1.72 \mathrm{eV}$ rather than in the one at
$1.53 \mathrm{eV}$, and the absence of RBLMs in the Raman spectrum excited at $1.72 \mathrm{eV}$ suggest that the $G$ modes' intensity in the spectrum excited at $1.72 \mathrm{eV}$ results from an outgoing resonance process in agreement with the presence of a mix of the $M_{11 \text {,in }}$ and $S_{33 \text {,out }}$ transitions around $1.53 \mathrm{eV}$. On the basis of the assignment of the transitions, we can understand the behavior of the REPs measured in the $1.45-1.70 \mathrm{eV}$ range.

The REP of the in-phase and out-of-phase RBLM is displayed in Fig. 8(a). Both REPS are well fitted by considering a single transition located around $1.53 \mathrm{eV}$, in agreement with the assignment of the $1.53 \mathrm{eV}$ transition as a a mix of the $M_{11-\text {,in }}$ and $S_{33 \text {,out }}$ transitions. The absence of interference effects in the profile of the out-of-phase RBLM seems to be opposite to the results of Bussi and collaborators which show a distortion of the REP of the RBM assigned to interference between the $M_{11-}$ and $M_{11+}$ transitions in metallic SWNTs [3]. However, the calculations of V.N. Popov et al. [6] predict a vanishing of the amplitude of the REP for the $M_{i i+}$ transition of the $(15,9)$ SWNT (see Fig. 2 of Ref. [6]). This explains the absences of the out-of-phase RBLM signal at $1.57 \mathrm{eV}$ and of interference effects in this particular case.

The REP of the $\mathrm{LO}_{\text {in }}$ mode of the inner metallic tube, located around $1558 \mathrm{~cm}^{-1}$, is displayed in Fig. 8(b) (green open dots). In this figure are compared the fits of the REP 
with interference between the $M_{11+\text {,in }}$ and $M_{11-\text {,in }}$ transitions [Fig. 8(b), black solid line] or without interference [Fig. 8(b), orange solid line]. The result of the fit of the REP of the $\mathrm{LO}_{\text {in }}$ mode by considering a single transition at $1.53 \mathrm{eV}$ (as in the fit of the REP of the RBLMs) is also displayed [Fig. 8(b), blue solid line]. Clearly, the REP of the $\mathrm{LO}_{\text {in }}$ mode is only well described when interferences between $M_{11-\text {,in }}$ and $M_{11+\text {,in }}$ transitions are taken into account [Fig. 8(b), black solid line]. The main effect of interferences is to distort the REP of the $\mathrm{LO}_{\text {in }}$ mode leading to a broad line shape centered at $1.55 \mathrm{eV}$. By contrast, the REPs of the $1558 \mathrm{~cm}^{-1}$ component (strongly dominated by $\mathrm{LO}_{\text {out }}$ mode) and $\mathrm{TO}_{\text {out }}$ mode are well described by considering a single transition at $1.53 \mathrm{eV}$ [Figs. 8(c) and 8(d), respectively).

\section{CONCLUSION}

In summary, combining optical absorption and Raman scattering on individual index identified DWNTs allows us to analyze and understand the effects of the quantum interference between different electronic transitions in the dependence of the intensity and profile of the longitudinal optical (LO) and transverse optical (TO) $G$ modes of the constituent inner and outer single-walled carbon nanotubes as a function of the excitation energy. In addition, the excitation dependence of each $G$ mode permits us to unambiguously assign each of them as originating from the $\mathrm{LO}$ or TO $G$ modes of inner and outer tubes. Finally, it must be emphasized that such effects can only be evidenced from experiments performed on individual index identified DWNTs.

\section{ACKNOWLEDGMENTS}

The authors thank Prof. A. San Miguel, Dr. J.-R. Huntzinger, and Dr. T. Michel for helpful discussions. We acknowledge Dr. T. X. Tinh for the synthesis of individual nanotubes. H.N.T., R.P., A.A.Z., J.L.S., and M.P. acknowledge financial support by the ANR GAMBIT project, Grant No. ANR-13-BS10-0014 of the French Agence Nationale de la Recherche, and the International project of Scientific Cooperation with Vietnam (CNRS PICS 6457). J.-C.B., A.A., F.V., and N.D.F. acknowledge financial support by ANR programme P2N under Contract ANR-11-NANO-025. The TEM studies were conducted at the Laboratorio de Microscopias Avanzadas, Instituto de Nanociencia de Aragon, Universidad de Zaragoza, Spain. Some of the research leading to these results has received funding from the European Union Seventh Framework Programme under Grant Agreement No. 312483- ESTEEM2 (Integrated Infrastructure Initiative I3). R.A. gratefully acknowledges the support from the Spanish Ministerio de Economia y Competitividad [FIS2013-46159C3-3-P and MAT2016-79776-P (AEI/FEDER, UE)] and from the Government of Aragon and the European Social Fund under the project "Construyendo Europa desde Aragon" 20142020 (Grant No. E/26).
[1] M. S. Dresselhaus, G. Dresselhaus, R. Saito, and A. Jorio, Annu. Rev. Phys. Chem. 58, 719 (2007).

[2] R. Saito, G. Dresselhaus, and M. S. Dresselhaus, Phys. Rev. B 61, 2981 (2000).

[3] G. Bussi, J. Menéndez, J. Ren, M. Canonico, and E. Molinari, Phys. Rev. B 71, 041404 (2005).

[4] J. G. Duque, H. Telg, H. Chen, A. K. Swan, A. P. Shreve, X. Tu, M. Zheng, and S. K. Doorn, Phys. Rev. Lett. 108, 117404 (2012).

[5] C. Thomsen, S. Reich, M. Cardona, and R. Merlin (eds.), Light Scattering in Solid IX (Springer, Berlin Heidelberg, 2007); Top. Appl. Phys. 108, 115 (2007), and references therein.

[6] V. N. Popov and P. Lambin, Phys. Rev. B 73, 165425 (2006).

[7] J.-C. Blancon, M. Paillet, H. N. Tran, X. T. Than, S. A. Guebrou, A. Ayari, A. San Miguel, N.-M. Phan, A. A. Zahab, J.-L. Sauvajol, N. Del Fatti, and F. Vallée, Nat. Commun. 4, 2542 (2013).

[8] K. Liu, X. Hong, M. Wu, F. Xiao, W. Wang, X. Bai, J. W. Ager, S. Aloni, A. Zettl, E. Wang, and F. Wang, Nat. Commun. 4, 1375 (2013).

[9] D. Levshov, T. X. Than, R. Arenal, V. N. Popov, R. Parret, M. Paillet, V. Jourdain, A. A. Zahab, T. Michel, Y. I. Yuzyuk, and J.-L. Sauvajol, Nano Lett. 11, 4800 (2011).

[10] V. N. Popov and L. Henrard, Phys. Rev. B 65, 235415 (2002).

[11] H. N. Tran, Phd thesis, University of Montpellier, France (2015).

[12] D. Levshov, T. Michel, R. Arenal, H. N. Tran, T. X. Than, M. Paillet, Yu. I. Yuzyuk, and J.-L. Sauvajol, J. Phys. Chem. C 119, 23196 (2015).

[13] J.-C. Blancon (private communication).
[14] K. Liu, C. Jin, X. Hong, J. Kim, A. Zettl, E. Wang, and F. Wang, Nat. Phys. 10, 737 (2014).

[15] S. Zhao, T. Kitagawa, Y. Miyauchi, K. Matsuda, H. Shinohara, and R. Kitaura, Nano Res. 7, 1548 (2014).

[16] T. X. Than, V. C. Nguyen, V. Jourdain, M. Paillet, D.-Y. Kim, J.-L. Sauvajol, T. T. T. Ngo, and N. M. Phan, J. Exp. Nanosci. 6, 547 (2011).

[17] R. Arenal, P. Loethman, M. Picher, X. T. Than, M. Paillet, and V. Jourdain, J. Phys. Chem. C 116, 14103 (2012).

[18] D. Levshov, H. N. Tran, R. Arenal, T. X. Than, M. Paillet, A. A. Zahab, Yu. I. Yuzyuk, J.-L. Sauvajol, and T. Michel, Carbon 114, 141 (2017).

[19] D. Christofilos, J.-C. Blancon, J. Arvanitidis, A. San Miguel, A. Ayari, N. Del Fatti, and F. Vallée, J. Phys. Chem. Lett. 3, 1176 (2012).

[20] H. N. Tran, J.-C. Blancon, J.-R. Huntzinger, R. Arenal, V. N. Popov, A. A. Zahab, A. Ayari, A. San-Miguel, F. Vallée, N. Del Fatti, J.-L. Sauvajol, and M. Paillet, Phys. Rev. B 94, 075430 (2016).

[21] K. Liu, J. Deslippe, F. Xiao, R. B. Capaz, X. Hong, S. Aloni, A. Zettl, W. Wang, X. Bai, S. G. Louie, E. Wang, and F. Wang, Nat. Nanotechnol. 7, 325 (2012).

[22] M. Paillet, T. Michel, J. C. Meyer, V. N. Popov, L. Henrard, S. Roth, and J.-L. Sauvajol, Phys. Rev. Lett. 96, 257401 (2006).

[23] H. Telg, J. G. Duque, M. Staiger, X. Tu, F. Hennrich, M. M. Kappes, M. Zheng, J. Maultzsch, C. Thomsen, and S. K. Doorn, ACS Nano 6, 904 (2012).

[24] T. Michel, M. Paillet, D. Nakabayashi, M. Picher, V. Jourdain, J. C. Meyer, A. A. Zahab, and J.-L. Sauvajol, Phys. Rev. B 80, 245416 (2009). 